\title{
Resiliencia, bienestar subjetivo y actitudes de los adolescentes hacia el consumo de drogas en Angola
}

\author{
Melchor Gutiérrez $^{1 *}$ e Isabel Romero ${ }^{2}$ \\ 1 Universidad de Valencia (España) \\ 2 Universidade Katyavala Bwila (Angola)
}

\begin{abstract}
Resumen: El objetivo del presente trabajo fue analizar las relaciones entre autoestima, inteligencia emocional, apoyo social, resiliencia, satisfacción con la vida y actitudes hacia el consumo de drogas de los adolescentes. Participaron 2506 adolescentes (1231 varones y 1275 mujeres) de edades comprendidas entre 14 y 18 años $(M=16.40 ; D T=1.40)$. Se utilizó un Modelo de Ecuaciones Estructurales para analizar los efectos de la autoestima, el apoyo social y la inteligencia emocional sobre la resiliencia, y de ésta sobre la satisfacción con la vida y las actitudes hacia el consumo de drogas de los adolescentes. Los resultados mostraron efectos positivos de la autoestima, el apoyo de la familia y el control emocional sobre la satisfacción con la vida de los adolescentes; y del uso de las emociones sobre la influencia de la familia para evitar el consumo de drogas. Por otro lado, fueron negativos los efectos del apoyo de la escuela, la evaluación de las emociones propias y la evaluación de las emociones de otros sobre la resiliencia; y de ésta sobre la satisfacción con la vida, la presión social y el efecto placentero de las drogas. Se discuten los resultados en el marco de la psicología positiva.

Palabras clave: Autoestima; apoyo social; inteligencia emocional; satisfac-
\end{abstract} ción con la vida; adolescentes; alcohol; tabaco; psicología positiva.
Title: Resilience, subjective well-being and adolescents' attitudes toward drug use in Angola

Abstract: The aim of this study was to analyse the relationship among self-esteem, emotional intelligence, social support, resilience, satisfaction with life and adolescents' attitudes toward drug use. Participants were 2506 adolescents (1231 males and 1275 females) aged from 14 to 18 years old $(M=16.40 ; S D=1.40)$. Structural Equation Modeling was carried out to examine de effects of self-esteem, social support and emotional intelligence on resilience, and the effect of resilience on adolescents' satisfaction with life and attitudes toward drug use. Results showed positive effects of self-esteem, family support and regulation of emotion on adolescents' satisfaction with life; and a positive effect of use of emotion on family influence to avoid adolescents' drug use. Additionally, the effects of school support, self-emotion appraisal and others' emotion appraisal on resilience were negative; as well as the effects of resilience on adolescents' satisfaction with life and drug use pleasure. Results are discussed in the positive psychology framework.

Key words: Self-esteem; social support; emotional intelligence; satisfaction with life; adolescents; alcohol; tobacco; positive psychology.

\section{Introducción}

Tradicionalmente, los campos de la psicología y la educación han destacado la identificación y forma de remediar los déficits de los adolescentes. Sin embargo, con la aparición de la psicología positiva (Seligman y Csikszentmihalyi, 2000; Sheldon y King, 2001), el foco de atención se comenzó a situar más en sus fortalezas, en las dimensiones positivas de su desarrollo psicosocial, con énfasis en la creación de ambientes óptimos que favorezcan su bienestar físico y psicológico (Alvarado y Ricard, 2013; Elmore y Huebner, 2010; Gutiérrez y Gonçalves, 2013; Oliva, Antolín y López, 2012; Oliva et al., 2010). Estudios realizados por Wood, Froh y Geraghty (2010) y Ho, Cheung y Cheung (2010) indicaron que el bienestar era el motor del desarrollo humano, y establecieron una relación entre el sentido de la vida, el optimismo y la calidad de vida de las personas. Sun y Shek (2012) encontraron que los adolescentes con un alto nivel de desarrollo positivo estaban más satisfechos con la vida y tenían menos problemas de conducta, con alto nivel de satisfacción y bajo nivel de problemas de conducta influyéndose entre sí.

Aunque es evidente esta tendencia actual a potenciar en la investigación el conocimiento de las fortalezas más que las debilidades, no por ello se puede ignorar la afición de muchos adolescentes por el consumo de sustancias adictivas,

* Dirección para correspondencia [Correspondence address] Melchor Gutiérrez. Universidad de Valencia. Facultad de Psicología. Dpto. de Psicología Evolutiva y de la Educación. Av. Blasco Ibáñez, 21. 46010 Valencia (España). E-mail: Melchor.Gutierrez@uv.es conducta contrapuesta al bienestar y que puede afectar su salud física y mental (Alfonso, Huedo y Espada, 2009; García del Castillo, Dias, Díaz-Pérez y García del Castillo-López, 2012; López y Rodríguez-Arias, 2010; Pérez y Lucio-Gómez, 2010; Ruiz y Ruiz-Risueño, 2011; Salamó, Gras y FontMayolas, 2010). Por ello, a lo largo de esta fundamentación teórica argumentaremos conjuntamente en favor de un desarrollo positivo y en la búsqueda de recursos para contravenir las conductas negativas, susceptibles de perjudicar la calidad de vida de los adolescentes. Partimos de la existencia de un razonable acuerdo internacional en considerar la calidad de vida como un constructo multidimensional que integra aspectos de salud, emocionales y de bienestar social percibidos por la persona (WHOQOL Group, 1995).

El bienestar subjetivo incluye juicios cognitivos tales como satisfacción con la vida, y respuestas emocionales ante los diferentes eventos como sentimiento de emociones positivas (Pavot y Diener, 2008). A lo largo de las tres últimas décadas, este constructo ha sido objeto de creciente interés entre los teóricos de diferentes orientaciones, tanto sociológicas como psicológicas, considerando la satisfacción con la vida como uno de sus elementos importantes (Diener, 2000; Marques, Pais-Ribeiro y Lopez, 2007; Rodríguez y Goñi, 2011). La satisfacción con la vida se define como la percepción subjetiva de la calidad de vida de una persona basada en las preferencias individuales de múltiples dominios de la vida y de la satisfacción en esos dominios. Goza de gran consideración en el campo de la psicología positiva porque está íntimamente relacionada con un amplio rango de beneficios personales, conductuales, psicológicos y sociales (Henrich y 
Herschbach, 2000; Liu, Wang y Lü, 2013; Lyubomirsky, King y Diener, 2005). Además, este constructo está considerado como una variable psicológica que puede ampliar nuestra comprensión de las dificultades de los adolescentes y del afrontamiento ante determinados retos del desarrollo (Terry y Huebner, 1995) como el consumo de sustancias adictivas. Los adolescentes con altos niveles de satisfacción con la vida tienen, en general, temperamento extrovertido, locus de control interno, activa capacidad de afrontamiento, autoconcepto positivo y perspectivas de futuro, así como tendencia a participar en actividades significativas de contenido prosocial. Por el contrario, niveles bajos de satisfacción con la vida se relacionan con resultados negativos tales como la conducta agresiva (Valois, Zullig, Huebner y Drane, 2001) y el abuso de sustancias adictivas (Zullig, Valois, Huebner, Oeltman y Drane, 2001).

La mayor parte de la investigación sobre bienestar subjetivo en general y satisfacción con la vida en particular, se ha desarrollado en población adulta, y han sido menos los trabajos destinados a analizar la satisfacción con la vida en niños y adolescentes (Gadermann, Schonert-Reichl y Zumbo, 2010). Además, dado que el bienestar subjetivo ha sido consistentemente identificado como un significativo factor psicológico asociado con el crecimiento positivo, la salud y el bienestar, es importante incrementar la comprensión de la satisfacción con la vida en adolescentes porque permite a los investigadores relacionar el bienestar subjetivo con características del desarrollo contemporáneo tales como el ajuste social y la salud física y mental (Overle, Schonert-Reichl y Zumbo, 2011). Teniendo en cuenta la limitada investigación que existe en este sentido, hemos diseñado el presente trabajo para ampliar la comprensión del bienestar subjetivo en la adolescencia, analizando la importancia de recursos personales y sociales positivos tales como la autoestima, el apoyo social, la inteligencia emocional y la resiliencia sobre el bienestar de los adolescentes, entendido éste como la satisfacción con la vida junto a las actitudes de evitación del consumo de drogas (desarrollo saludable).

Los investigadores han encontrado un importante número de características y recursos que pueden influir en la protección contra los riesgos del uso de sustancias, tanto a nivel individual como de los amigos, la familia, la escuela o la comunidad, y favorecer el bienestar psicológico (Panadero y Muñoz, 2014; Sánchez y Barrón, 2003). Entre tales recursos, uno importante es la autoestima (Byrne y Mazanov, 2001; Richardson, Kwon y Ratner, 2013; Videra y Reigal, 2013), entendida como la evaluación que el individuo hace de sí mismo. La autoestima expresa una actitud de aprobación o de repulsa de sí y engloba el auto-juicio en relación a competencia y valor. Este juicio afecta a la manera en que el individuo se relaciona o desenvuelve en esferas como la familiar, la social y la académica (Avanci, Assis, Santos y Oliveira, 2007; Buelga, Cava y Musitu, 2012). Una baja autoestima se caracteriza por el sentimiento de incompetencia, de inadecuación a la vida y de incapacidad para superar desafíos, mientras que la autoestima elevada expresa un sentimiento de confianza y competencia (Branden, 2000). Tener baja autoestima puede colocar a un adolescente en riesgo para desarrollar resultados no deseables. Tener una alta autoestima, por el contrario, puede ser una cualidad que puede proteger al joven de los resultados negativos asociados con la exposición al riesgo (Fergus y Zimmerman, 2005).

En la última década, numerosos estudios se han centrado en el papel que la autoestima puede jugar en relación con la promoción de conductas saludables o con la evitación de las no saludables (Veselska et al., 2009). Se ha encontrado una asociación de esta variable con la iniciación y el consumo de tabaco y otras sustancias adictivas (Kokkevi, Richardson, Florescu, Kuzman y Stergar, 2007; Richardson et al., 2013; Wild, Flisher, Bhana y Lombard, 2004). La autoestima positiva o elevada resulta esencial en el desarrollo mental saludable así como factor protector de conductas sociales inadecuadas. Por el contrario, la autoestima negativa o baja autoestima puede jugar un papel importante en el desarrollo de un amplio rango de desórdenes mentales y problemas sociales tales como depresión, ansiedad, violencia, conductas de riesgo y uso de sustancias adictivas (Buelga et al, 2012; Mann, Hosman, Schaalma y de Vries, 2004).

Las repercusiones del consumo de alcohol y tabaco, sobre todo en la etapa de crecimiento, son considerables, provocando consecuencias tanto físicas como psicológicas. Por ello, son numerosos los estudios que tratan de detectar los posibles factores de protección y riesgo de consumo, encontrándose, entre otros, rasgos de personalidad, baja autoestima, locus de control externo, baja tolerancia a la frustración, factores cognitivos, actitudes, creencias y expectativas (Alfonso et al., 2009; Ruiz y Ruiz-Risueño, 2011). Para comprender mejor cómo los adolescentes se enfrentan a los problemas de salud, sin duda es importante identificar los factores que pueden promover la resiliencia, tales como el optimismo, el afrontamiento activo y el apoyo social (Braverman, 1999; Liu et al., 2013; Smith et al., 2008). Haggerty, Sherrod, Gamezy y Rutter (2000) y Sapienza y Pedromônico (2005) destacaron que el apoyo social y la autoestima sirven de protección contra los efectos de experiencias estresantes.

Son varias las interpretaciones que últimamente se viene haciendo de la resiliencia. Puede entenderse como proceso de, capacidad para, o resultado de la adaptación exitosa frente a circunstancias desafiantes o amenazantes; es la capacidad para recuperarse y mantener una conducta adaptativa después de sufrir un estrés intenso o experiencias traumáticas. También es considerada como una característica de personalidad positiva que mejora la adaptación individual. Generalmente, la resiliencia se refiere al proceso de superar los efectos negativos de la exposición al riesgo, afrontar con éxito experiencias traumáticas y evitar trayectorias negativas asociadas con riesgos. La teoría de la resiliencia relacionada con la exposición a riesgos de los adolescentes, se centra más en las fortalezas que en los déficits (psicología positiva), en la comprensión del desarrollo de la salud a pesar de la exposición al riesgo (Fergus y Zimmerman, 2005). Los adolescentes resilientes tienen entre ellos mismos, su familia, su grupo de 
iguales y su entorno, factores de protección que les ayudan a defenderse ante las fuerzas negativas y estresantes a las que están expuestos en su vida diaria (Boyce Rodgers y Rose, 2002; Liu et al., 2013). Otros estudios (Buckner, Mezzacappa y Beardslee, 2003; Gordon Rouse, Ingersoll y Orr, 1998) han encontrado que los adolescentes resilientes tenían mayor autoestima y era menos probable que se vieran implicados en conductas de riesgo en comparación con sus iguales menos resilientes. Según Becoña, Mínguez, López, Vázquez y Lorenzo (2006), los adolescentes que no consumían alcohol tenían una mayor puntuación en resiliencia.

Otro factor que influye positivamente sobre el desarrollo de los adolescentes es el apoyo social, entendido como la relación humana en que se intercambian recursos socioemocionales (comprensión, consuelo, aceptación, consejo), instrumentales (bienes y servicios en tareas de la vida cotidiana) o recreativas (compañía en diversión o recreación). No es un fenómeno simple, se presenta como un conjunto de factores en interacción. Es dinámico, cambia con el tiempo y a medida que cambian las situaciones de la vida. Por tanto, el apoyo social se ha definido como el conjunto de actividades y comportamientos, relaciones, interacciones y cualidades de las relaciones sociales (Cohen, 2004). La familia, los amigos, los vecinos, componen el sistema de apoyo social informal y constituyen las principales fuentes de apoyo de los adolescentes (Feitosa, de Matos, del Prette y del Prette, 2005). Contar con un buen apoyo social favorece la percepción de satisfacción con la vida, de tener alta autoestima y bienestar psicológico, y a su vez tener una actitud no violenta (Clara, Cox, Enns, Murray y Torgrudc, 2003). Está ampliamente documentado que el apoyo social se encuentra asociado con la salud y el bienestar y que constituye un buen aliado para mejorar el afrontamiento de los problemas de salud y conductas negativas (Ben-Ari y Gil, 2004).

Diversas investigaciones han demostrado el impacto de los padres, amigos, profesores y compañeros de clase tanto sobre las conductas conflictivas, problemas emocionales y desajuste escolar, como en favor del desarrollo positivo de los adolescentes (Bokhorst, Sumter y Westenberg, 2010; Cook, Herman, Phillips y Settersten, 2002; Demaray y Malecki, 2002; Garnefski y Diekstra, 1996). Ya en 1985 Cohen y Wills afirmaban que el apoyo social está relacionado con el bienestar porque ofrece emociones positivas, sentido de valía personal y de predicción de la vida, y funciona como amortiguador del estrés, refuerzo de la autoestima, autoeficacia y capacidad de resolución de problemas, por lo que se interpreta que tiene efectos positivos sobre el bienestar. No obstante, aunque algunas investigaciones han explorado las asociaciones entre el apoyo social y el bienestar en los adultos, aún queda bastante por aclarar sobre las relaciones globales y las asociaciones entre los diferentes tipos de apoyo social y el bienestar en los adolescentes (Chu, Saucier y Hafner, 2010).

Así mismo, muy relacionado con el objeto de estudio que aquí nos ocupa está la inteligencia emocional (Liu et al., 2013; Skaar y Williams, 2012). Ésta se ha conceptualizado como un conjunto de habilidades concernientes a la regula- ción, gestión, control y uso de las emociones en la toma de decisiones (Mayer, Salovey y Caruso, 2008), y en relación con la promoción de la salud y el funcionamiento psicosocial adaptativo. La evaluación de la inteligencia emocional de los adolescentes ofrece una ventana para predecir, comprender y atenuar los problemas de conducta (Downey, Johnston, Hansen, Birney y Stough, 2010; Petrides, Frederickson y Furnham, 2004). Niveles elevados de inteligencia emocional han mostrado estar asociados con el bienestar subjetivo (Austin, Saklofske y Egan, 2005), mientras que bajos niveles de inteligencia emocional han sido predictores de conductas psicológicamente desajustadas. Más específicamente, la capacidad de gestión de las propias emociones está negativamente asociada con la manifestación de conductas problemáticas. Por ello, esta capacidad de gestionar las emociones se ha considerado el primer paso hacia la reducción de problemas de conducta, siendo a su vez un factor protector en la reducción de las emociones negativas (Schutte et al., 2010; Skaar y Williams, 2012).

Últimamente se ha incrementado la investigación en la que se viene prestando atención al papel que los procesos de autocontrol pueden jugar en la escalada del consumo de sustancias adictivas durante la adolescencia (Brody y Ge, 2001; Wills, Walker, Mendoza y Ainette, 2006; Wills y Dishion, 2004). Existe un importante cuerpo teórico sobre la regulación emocional en el que se sugiere la relevancia del autocontrol emocional en aspectos del desarrollo de los adolescentes tales como la competencia social; sin embargo, se le ha prestado menor atención a esta variable en la investigación sobre el uso de sustancias adictivas, a pesar de disponer de evidencias que sugieren que la regulación emocional puede ser un factor importante en el uso y abuso de sustancias por parte de los adolescentes (Simons y Carey, 2002). Los resultados de las investigaciones han mostrado que la regulación emocional es bastante relevante para predecir el uso de sustancias y sugieren que varios aspectos de la regulación de la emoción tienen componentes comunes que contribuyen a un mejor o peor control emocional (Wills y Stoolmiller, 2002; Wills et al, 2006). García del Castillo et al. (2012) y García del Castillo y Dias (2007) destacaron la importancia de la autorregulación, señalando que produce un efecto de coping, es decir, similar a la resiliencia, en cuanto a la resistencia a tomar alcohol o fumar.

Dado que existen planteamientos teóricos que defienden que las variables propias del desarrollo positivo de los adolescentes (por ejemplo su autoestima, apoyo social, inteligencia emocional y resiliencia, entre otros) influyen sobre el bienestar y salud de la persona (Ciarrochi, Scott, Deane y Heaven, 2003; Lent et al., 2005; Sun y Shek, 2010), y basados especialmente en los trabajos de Sun y Shek (2012), proponemos un modelo teórico para predecir conjuntamente la percepción de bienestar subjetivo (satisfacción con la vida) y las actitudes hacia el consumo de substancias adictivas de los adolescentes, a partir de su autoestima, el apoyo social y la inteligencia emocional, actuando como variable mediadora la resiliencia (Figura 1). Partimos de tres hipótesis: (1) Que la autoestima, el apoyo social y la inteligencia emocional de los adolescentes presentarán relaciones di- 
rectas con su resiliencia. (2) Que la resiliencia de los adolescentes se relacionará directamente con su bienestar subjetivo, e indirectamente con sus actitudes favorables hacia el consumo de drogas. (3) Que la satisfacción con la vida y las actitudes en favor del consumo de drogas correlacionarán de forma negativa.

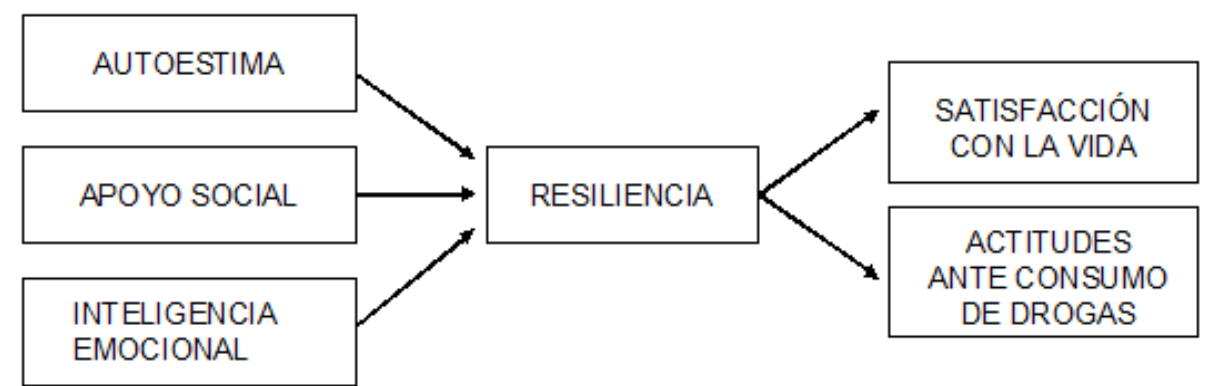

Figura 1. Modelo teórico para la predicción de la satisfacción con la vida y las actitudes de los adolescentes hacia el consumo de drogas.

\section{Método}

\section{Participantes}

Participaron en la investigación un total de 2506 adolescentes $(49.1 \%$ varones y $50.9 \%$ mujeres), de edades comprendidas entre 14 y 18 años $(M=16.40 ; D T=1.40)$, todos ellos estudiantes de diferentes centros educativos de tres provincias de Angola (Benguela, Huíla y Luanda) dispuestos a colaborar en el estudio. El procedimiento de muestreo ha sido incidental o por conveniencia, a través de colaboradores, procurando un equilibrio en variables sociodemográficas como la edad, el género y el nivel sociocultural, de cara a conseguir la mayor representatividad posible de la muestra en la población. La distribución de los participantes se expone en la Tabla 1.

Tabla 1. Distribución de la muestra según la edad y el género de los adolescentes.

\begin{tabular}{|c|c|c|c|c|c|c|}
\hline \multirow[b]{2}{*}{ Edad } & \multicolumn{2}{|c|}{ Varones } & \multicolumn{2}{|c|}{ Mujeres } & \multicolumn{2}{|c|}{ Total } \\
\hline & $n$ & $\%$ & $n$ & $\%$ & $n$ & $\%$ \\
\hline 14 años & 147 & 5.9 & 191 & 7.6 & 338 & 13.5 \\
\hline 15 años & 187 & 7.5 & 198 & 7.9 & 385 & 15.4 \\
\hline 16 años & 236 & 9.4 & 238 & 9.5 & 474 & 18.9 \\
\hline 17 años & 273 & 10.9 & 291 & 11.6 & 564 & 22.5 \\
\hline 18 años & 388 & 15.5 & 357 & 14.2 & 745 & 29.7 \\
\hline Total & 1231 & 49.1 & 1275 & 50.9 & 2506 & 100.0 \\
\hline
\end{tabular}

\section{Instrumentos}

Escala de Autoestima (RSES; Rosenberg, 1965). Es una escala formada originariamente por 10 items, cinco positivos y cinco invertidos (ejemplos: A veces me siento realmente inútil; Tengo una actitud positiva en relación a mí mismo). Ha sido traducida, adaptada y validada en numerosos idiomas y en más de 50 naciones. En la literatura especializada, hay autores que defienden su estructura unidimensional (Schmitt y Allik, 2005), y otros consideran que está formada por dos dimensiones, estima positiva y estima negativa (Avanci et al., 2007). Adicionalmente, también existen trabajos que indican que, debido a la forma de redacción de los ítems, unos en positivo y otros invertidos, se incorpora un artefacto estadístico o efecto de método (Santos y Maia, 2003; Tomás y Oliver, 1999), lo cual ha llevado a algunos autores a considerar únicamente los ítems redactados en positivo (Oliver, Sancho, Galiana, Tomás y Gutiérrez, 2012). Esta última es la opción que aquí hemos tomado, a partir de la versión portuguesa ofrecida por Avanci et al. (2007).

Todas las escalas aplicadas en la presente investigación se han presentado a los participantes con cinco alternativas de respuesta, desde (1) totalmente en desacuerdo, hasta (5) totalmente de acuerdo.

Escala de Apoyo Social (MSPSS-AA; Zimet, Dahlem, Zimet y Farley, 1988). Se ha utilizado la versión de Ramaswamy, Aroian y Templin (2009). Está compuesta por 12 ítems agrupados en tres factores: Apoyo del personal de la escuela, apoyo de los amigos, y apoyo de la familia (ejemplos: Tengo un profesor orientador que trata de ayudarme; Puedo hablar sobre mis problemas con mis amigos; Mi familia es realmente una fuente de confort para mî).

Escala de Inteligencia Emocional (Wong y Law, 2002). Consta de 16 ítems y mide cuatro dimensiones: Evaluación de las emociones propias, evaluación de las emociones de otros, uso de las emociones, y control emocional (ejemplos: Tengo buena percepción de mis propias emociones; Soy sensible a los sentimientos y emociones de otros; Soy una persona auto-incentivada; Soy bastante capaz de controlar mis propias emociones).

Escala de Resiliencia (Smith et al., 2008). Es una escala unidimensional y está formada por seis ítems (ejemplos: Tengo tendencia a desistir rápidamente ante las dificultades; No tardo mucho tiempo en recuperarme de un evento estresante). Para nuestros objetivos hemos utilizado únicamente cuatro ítems, con la intención de evitar el efecto de método de los ítems invertidos, similar al referido anteriormente en relación con la escala de autoestima.

Escala de Satisfacción con la Vida (SWLS; Pavot y Diener, 1993, 2008). Está compuesta por cinco ítems, cuyo objetivo es proporcionar un índice general de satisfacción con la vida 
referido a la percepción de bienestar subjetivo de los adolescentes (ejemplos: Estoy satisfecho con mi vida; En la mayoría de los aspectos mi vida es como quiero que sea).

Cuestionario de Actitudes hacia el Consumo de Drogas. Instrumento elaborado a partir de los trabajos de Escámez y García (1990) y de Ortega, Mínguez y Pagán (1993). Con este instrumento se pretende medir los efectos placenteros del consumo de drogas, la dependencia de las drogas, la presión social para el consumo de drogas, el perjuicio de las drogas para la salud, y la tolerancia/aceptación social ante el consumo de drogas (ejemplos: Las bebidas alcohólicas hacen que me sienta bien; Si fumara me podría dejar el tabaco cuando quisiera; Es conveniente fumar para no ser rechazado por los amigos; Prefiero no estar en lugares donde se consumen drogas; No es necesario probar las drogas para saber que perjudicarían mi salud).

\section{Procedimiento}

En primer lugar, se solicitó permiso a las autoridades del Ministerio de Educación de Angola para desarrollar la investigación. Seguidamente se contactó con las autoridades de los centros educativos que habían manifestado su disposición a colaborar, a los cuales se explicó en qué consistía la investigación y se les solicitó permiso para llevarla a cabo. Finalmente, se habló con los alumnos y se les facilitó una carta dirigida a los padres o tutores responsables de los menores de edad, solicitando permiso para su participación en el estudio. Todos los alumnos que participaron lo hicieron voluntariamente, tras devolver el consentimiento firmado de sus padres o tutores. Previo a la aplicación de los instrumentos, se procedió a traducirlos al portugués, excepto la escala de autoestima y la de satisfacción con la vida, de las cuales ya se contaba con versiones publicadas en ese idioma. La aplicación de los instrumentos se realizó en el horario habitual de clase, sin la presencia de los profesores, para garantizar en mayor medida el anonimato de las respuestas.

\section{Análisis de datos}

Los datos han sido analizados a tres niveles. Por una parte, se ha procedido a calcular las propiedades psicométricas de los instrumentos administrados a los participantes, con el objetivo de comprobar su validez y fiabilidad. Para ello, se han realizado diversos Análisis Factoriales Confirmatorios, basándose en modelos ya existentes, y comprobando así la validez de los instrumentos para ser aplicados a esta muestra. También se ha calculado la consistencia interna (coeficiente alpha de Cronbach) de los factores que componen cada uno de los instrumentos empleados en el estudio. Por otro lado, se han calculado las correlaciones entre las variables estudiadas. Finalmente, se ha desarrollado un Modelo de Ecuaciones Estructurales con variables observables (path análisis) puesto a prueba para calcular la predicción del bienestar subjetivo y las actitudes hacia el consumo de substancias adicti- vas de los adolescentes, así como el posible efecto mediador de la resiliencia.

Para llevar a cabo los análisis factoriales confirmatorios (AFCs; Bollen, 1989), se ha empleado el programa EQS 6.1 (Bentler y Wu, 1995). Los modelos confirmatorios se han estimado mediante máxima verosimilitud con correcciones robustas de Satorra-Bentler en los errores estándar y estadísticos e índices de ajuste, dada la falta de normalidad de las variables (Finney y DiStefano, 2006). El ajuste de los análisis factoriales confirmatorios se ha evaluado utilizando diversos criterios, tal como se recomienda en la literatura (Hu y Bentler, 1999). Concretamente, los estadísticos e índices de ajute utilizados son: (a) estadístico $\chi^{2}$ (Kaplan, 2000); (b) Comparative Fit Index (CFI; Bentler, 1990) mayor de .90 (e idealmente mayor de .95; Hu y Bentler, 1999); (c) Goodness-of-Fit Index (GFI), cuyos valores superiores a .90 indican un buen ajuste; (d) Standardized Root Mean Square Residual (SRMR), con valores menores de .06 considerados como indicadores de un buen ajuste; y (e) Root Mean Square Error of Approximation (RMSEA), cuyos valores son aceptables cuando son iguales o menores a .05. Como referencia, Hu y Bentler (1999) señalan que un conjunto de al menos .95 , junto con RMSEA de al menos .06, indican un buen ajuste del modelo hipotetizado a los datos.

\section{Resultados}

\section{Propiedades psicométricas de los instrumentos em- pleados}

Escala de Autoestima. Mediante AFCs, se sometieron a comprobación en nuestra muestra de estudio los tres modelos que ofrece la literatura: Un factor general, dos factores, y un factor más el efecto de método. Los resultados obtenidos indican que el primer modelo, según el cual se considera la autoestima como un factor global, muestra unos índices de ajuste a los datos considerados no satisfactorios $\left(\chi^{2}{ }_{35}=\right.$ $1120.71, p<.01, \mathrm{GFI}=.900, \mathrm{AGFI}=.842, \mathrm{CFI}=.517$, SRMR $=.098$, RMSEA $=.106)$. Mejores ajustes muestran los índices relativos al segundo modelo, en el que se concibe la Autoestima formada por un factor positivo y otro factor negativo $\left(x^{2}{ }_{34}=339.41, p<.01, \mathrm{GFI}=.972, \mathrm{AGFI}=.955\right.$, $\mathrm{CFI}=.864, \mathrm{SRMR}=.059, \mathrm{RMSEA}=.057)$. No obstante, los mejores índices de ajuste los ofrece la consideración de la autoestima formada por un factor de autoestima global más el efecto de método $\left(\chi^{2}{ }_{30}=149.05, p<.01\right.$, GFI $=.987$, $\mathrm{AGFI}=.976, \mathrm{CFI}=.947, \mathrm{SRMR}=.028, \mathrm{RMSEA}=.038)$.

A la vista de estos resultados, hemos optado por considerar para los presentes cálculos únicamente los ítems positivos de la escala. El coeficiente alfa de Cronbach (consistencia interna) de este factor es de .715 .

Escala de Apoyo Social. Sometida a un AFC la versión de la Escala de Apoio Social de Ramaswamy et al. (2009), se han obtenido satisfactorios índices de ajuste a los datos: $\chi^{2}{ }_{51}=$ 257.24, $p<.001$, GFI $=.965$, AGFI $=.946$, CFI $=.984$, 
SRMR $=.034$, RMSEA $=.038$, ratificándose los tres factores anunciados por sus autores: Apoyo social de la escuela $(\alpha=$ $.809)$, apoyo social de los amigos $(\alpha=.747)$, y apoyo social de la familia $(\alpha=.742)$.

Escala de Inteligencia Emocional. El AFC al que se sometió el modelo de Inteligencia Emocional ofrecido por Wong y Law (2002), ha aportado satisfactorios índices de ajuste a los datos: $\chi^{2} 98=377.33, p<.001, \mathrm{GFI}=.963, \mathrm{AGFI}=.949, \mathrm{CFI}$ $=.981, \mathrm{SRMR}=.038, \mathrm{RMSEA}=.032$, lo cual confirma la presencia de cuatro factores, tal como proponen sus autores. La consistencia interna de los factores que componen la Inteligencia Emocional es la siguiente: Evaluación de las emociones propias $(\alpha=.748)$, Evaluación de las emociones de otros $(\alpha=.729)$, Uso de las emociones $(\alpha=.784)$, y Control emocional $(\alpha=.725)$.

Escala de Resiliencia. El AFC de la Escala de Resiliencia construida a partir de la versión de Smith et al. (2008) ha proporcionado los siguientes índices de ajuste: $\chi^{2}{ }_{2}=0.45, p=$ $.79, \mathrm{GFI}=1.000, \mathrm{AGFI}=.999, \mathrm{CFI}=1.000, \mathrm{SRMR}=.004$, RMSEA $=.000)$, lo cual indica que los cuatro ítems se agrupan en una sola dimensión, con un coeficiente de consistencia interna de $\alpha=.705$. Dado que los ítems están todos redactados en negativo, se ha invertido el valor de los datos de tal manera que una mayor puntuación indique un mayor nivel de resiliencia.

Escala de Satisfacción con la Vida. La escala de Satisfacción con la Vida (SWLS, Pavot y Diener, 1993), cuya estructura unidimensional ha sido ya ratificada por numerosos autores en diferentes culturas, muestra aquí esta misma estructura con un buen ajuste del modelo a los datos: $\chi^{2}{ }_{5}=25.26, p<$ .001 , GFI $=.993$, AGFI $=.979, \mathrm{CFI}=.990, \mathrm{SRMR}=.023$, RMSEA $=.038$. Su consistencia interna es de $\alpha=.735$

Cuestionario de Actitudes hacia el Consumo de Drogas. Dado que la literatura especializada no aporta datos de AFC relativos a este cuestionario, se sometió a prueba el modelo conceptual ofrecido por sus autores (Ortega, et al., 1993). Para ello, se dividió la muestra de adolescentes en dos submuestras al azar, con el 50\% de participantes en cada una de ellas, y se comenzó probando el modelo en una de las submuestras, obteniendo los siguientes índices de ajuste: $\chi^{2} 220=$ 1584.81, $p<.001$, GFI $=.875$, AGFI $=.844$, CFI $=.761$, $\mathrm{SRMR}=.094, \mathrm{RMSEA}=.068$. Estos resultados indican que el ajuste del modelo para los datos de nuestra muestra es pobre. Eliminados los 5 ítems que no aportaban una saturación satisfactoria, el ajuste fue mucho más adecuado: $\chi^{2}{ }_{125}=$ $312.45, p<.001$, GFI $=.969$, AGFI $=.957$, CFI $=.956$, SRMR $=.033$, RMSEA $=.033$. Seguidamente, el modelo obtenido con la primera submuestra se sometió a validación en la segunda submuestra, y aportó unos índices de ajuste muy satisfactorios: $\chi^{2} 125=219.97, p<.001, \mathrm{GFI}=.978, \mathrm{AGFI}=$ $.970, \mathrm{CFI}=.978, \mathrm{SRMR}=.029, \mathrm{RMSEA}=.023$.

Los coeficientes de consistencia interna de los cinco factores extraídos del Cuestionario de Actitudes hacia el Consumo de Drogas fueron: Las drogas no perjudican la salud ( $\alpha$ $=.730)$, presión social para el consumo de drogas $(\alpha=.803)$, las drogas no generan dependencia $(\alpha=.772)$, efecto placentero del consumo de drogas $(\alpha=.731)$, influencia de la familia para evitar el consumo de drogas $(\alpha=.716)$. Las valoraciones de los ítems han sido consideradas en el mismo sentido que el título de los factores que los agrupan. Así, por ejemplo, una mayor puntuación indicaría que los adolescentes consideran que las drogas no perjudican la salud; y a mayor puntuación, mayor es considerado por los adolescentes el efecto placentero del consumo de drogas. Asimismo, una mayor puntuación indicaría un mayor poder de influencia de la familia para evitar el consumo de drogas.

\section{Estadísticos descriptivos y correlaciones entre las variables estudiadas}

De los resultados mostrados en la Tabla 2 cabe destacar un moderado nivel de autoestima de los adolescentes $(M=$ $3.84, D T=0.71)$, una considerable percepción de apoyo social de los amigos $(M=3.98, D T=0.81)$ y de la familia $(M=$ 3.97, $D T=0.80)$, así como capacidad para la evaluación de las emociones propias $(M=3.82, D T=0.73)$. Por otro lado, también cabe señalar el bajo nivel de resiliencia manifestado por los adolescentes $(M=2.87, D T=0.84)$, el desacuerdo con que las drogas no perjudican la salud $(M=2.00, D T=$ $0.86)$, la negativa a reconocer la presión social para el consumo de drogas $(M=1.68, D T=0.76)$, y la resistencia a reconocer el efecto placentero del consumo de drogas $(M=$ 1.91, DT = 0.74).

Con respecto a las correlaciones entre las variables estudiadas, se ha encontrado que la autoestima correlaciona positivamente con los factores del apoyo social (valores de $r$ entre .33 y $.35, p<.01)$, con los factores de la inteligencia emocional ( $r$ entre .22 y .33, $p<.01)$, con la satisfacción con la vida $(r=.32, p<.01)$, y con la influencia de la familia en el consumo de drogas $(r=.18, p<.01)$, mientras que las correlaciones son negativas con "las drogas no perjudican la salud" $(r=-.12, p<.01)$, con la "presión social para el consumo de drogas" $(r=-.14, p<.01)$ y con el "efecto placentero del consumo de drogas" $(r=-.12, p<.01)$. Así mismo, cabe destacar la falta de relación entre la autoestima y la resiliencia $(r=-.03, p>.05)$ y las correlaciones negativas entre la resiliencia y las demás variables estudiadas, tanto del apoyo social como de la inteligencia emocional y de las actitudes hacia el consumo de drogas de los adolescentes. Finalmente, la satisfacción con la vida de los adolescentes se encuentra positivamente relacionada, además de con la autoestima ya mencionada, con el apoyo social (valores de $r$ entre .21 y $.35, p<$ .01 ) y con la inteligencia emocional (valores de $r$ entre $.15 \mathrm{y}$ $.28, p<.01)$. Por el contrario, correlaciona negativamente con la resiliencia $(r=-.12, p<.01)$. De las relaciones encontradas entre la satisfacción con la vida y las actitudes hacia la droga, ninguna de ellas resulta ser estadísticamente significativa. 
Tabla 2. Medias, desviaciones típicas, alfas y correlaciones bivariadas entre las variables estudiadas.

\begin{tabular}{|c|c|c|c|c|c|c|c|c|c|c|c|c|c|c|c|}
\hline Variables & 1 & 2 & 3 & 4 & 5 & 6 & 7 & 8 & 9 & 10 & 11 & 12 & 13 & 14 & 15 \\
\hline $1 . \mathrm{AE}$ & 1 & $.20 * *$ & $.35^{* *}$ & $.35^{* *}$ & $.33^{* *}$ & $.22^{* *}$ & $.37 * *$ & $.26^{* *}$ & -.03 & $.32^{* *}$ & $-.12 * *$ & $-.14 * *$ & -.01 & $-.12 * *$ & $.18^{* *}$ \\
\hline 2. ApSE & & 1 & $.15^{* *}$ & $.26^{* *}$ & $.16^{* *}$ & $.09 * *$ & $.11 * *$ & $.18^{* *}$ & $-.16^{* *}$ & $.21 * *$ & $.06 * *$ & $.08 * *$ & -.01 & .01 & -.01 \\
\hline 3. ApSA & & & 1 & $.31 * *$ & $.23^{* *}$ & $.23^{* *}$ & $.24^{* *}$ & $.14^{* *}$ & $-.05^{*}$ & $.21 * *$ & $-.11^{* *}$ & $-.15^{* *}$ & .03 & $-.06 * *$ & $.15^{* *}$ \\
\hline 4. ApSF & & & & 1 & $.24 * *$ & $.16^{* *}$ & $.23^{* *}$ & $.22 * *$ & $-.05^{*}$ & $.35^{* *}$ & $-.08^{* *}$ & $-.07 * *$ & .02 & $-.12 * *$ & $.11^{* *}$ \\
\hline 5. EEP & & & & & 1 & $.35^{* *}$ & $.39 * *$ & $.40^{* *}$ & $-.11 * *$ & $.25^{* *}$ & $-.07 * *$ & $-.08^{* *}$ & $.08^{* *}$ & $-.06 * *$ & $.20^{* *}$ \\
\hline 6. EEO & & & & & & 1 & $.36^{* *}$ & $.28^{* *}$ & $-.12^{* *}$ & $.15^{* *}$ & -.02 & $-.05^{*}$ & $.06^{* *}$ & -.01 & $.17^{* *}$ \\
\hline 7. UEm & & & & & & & 1 & $.39 * *$ & $-.08^{* *}$ & $.24 * *$ & $-.06 * *$ & $-.09 * *$ & .03 & $-.09 * *$ & $.21 * *$ \\
\hline 8. CEm & & & & & & & & 1 & $-.08^{* *}$ & $.28^{* *}$ & .01 & .02 & .03 & $-.05^{*}$ & $.06^{* *}$ \\
\hline 9. RES & & & & & & & & & 1 & $-.12^{* *}$ & $-.17 * *$ & $-.14 * *$ & -.02 & $-.13^{* *}$ & -.03 \\
\hline 10. SatV & & & & & & & & & & 1 & .04 & .04 & .03 & -.01 & .03 \\
\hline 11. DNPS & & & & & & & & & & & 1 & $.52^{* *}$ & $.15^{* *}$ & $.44^{* *}$ & -.03 \\
\hline 12. PSCD & & & & & & & & & & & & 1 & $.16^{* *}$ & $.55^{* *}$ & -.04 \\
\hline 13. DNGD & & & & & & & & & & & & & 1 & $.20^{* *}$ & $.14^{* *}$ \\
\hline 14. EPCD & & & & & & & & & & & & & & 1 & .01 \\
\hline 15. IFECD & & & & & & & & & & & & & & & 1 \\
\hline$M$ & 3.84 & 3.33 & 3.98 & 3.97 & 3.82 & 3.51 & 3.79 & 3.67 & 2.87 & 3.39 & 2.00 & 1.68 & 2.89 & 1.91 & 3.60 \\
\hline D.T. & 0.71 & 1.05 & 0.81 & 0.80 & 0.73 & 0.79 & 0.72 & 0.83 & 0.84 & 0.78 & 0.86 & 0.76 & 1.31 & 0.74 & 0.99 \\
\hline Alfa & .715 & .809 & .747 & .742 & .748 & .729 & .784 & .725 & .705 & .753 & .730 & .803 & .772 & .731 & .716 \\
\hline
\end{tabular}

Notas. $* \mathrm{p}<.05 ; * * \mathrm{p}<.01 ;$ AE: Autoestima; ApSE: Apoyo social de la escuela; ApSA: Apoyo social de los amigos; ApSF: Apoyo social de la familia; EEP: Evaluación de las emociones propias; EEO: Evaluación de las emociones de otros; UEm: Uso de la emoción; CEm: Control emocional; RES: Resiliencia; SatV: Satisfacción con la vida; DNPS: Las drogas no perjudican la salud; PSCD: Presión social para el consumo de drogas; DNGD: Las drogas no generan dependencia; EPCD: Efecto placentero del consumo de drogas; IFECD: Influencia de la familia para evitar el consumo de drogas.

Predicción del bienestar subjetivo y las actitudes de los adolescentes hacia el consumo de drogas

El modelo hipotético inicial planteaba que la autoestima, el apoyo social y la inteligencia emocional, estarían positivamente relacionados con la resiliencia, la cual, a su vez, tendría capacidad predictiva sobre la satisfacción con la vida y las actitudes hacia el consumo de drogas de los adolescentes. Este modelo general a-priori se puso a prueba mediante ecuaciones estructurales, obteniendo los siguientes índices de ajuste: $\chi^{2}{ }_{53}=1059.54, p<.01, \mathrm{GFI}=.951, \mathrm{AGFI}=.888, \mathrm{CFI}$ $=.819, \mathrm{SRMR}=.088, \mathrm{RMSEA}=.083$. Dado que tales resultados no pueden considerarse satisfactorios, se probó un nuevo modelo, en el que se eliminaron las relaciones no significativas, obteniendo unos índices de ajuste a los datos mucho más satisfactorios: $\chi^{2}{ }_{57}=390.64, p<.01$, GFI $=.978$, $\mathrm{AGFI}=.953, \mathrm{CFI}=.932, \mathrm{SRMR}=.048, \mathrm{RMSEA}=.048$.

Los resultados de este segundo modelo (Figura 2) muestran que la autoestima se encuentra positiva y directamente relacionada con la resiliencia y la satisfacción con la vida de los adolescentes. Por otro lado, el apoyo social de la escuela se relaciona negativamente con la resiliencia, el apoyo de los amigos no presenta relaciones significativas con ninguna de las variables, y el apoyo de la familia se encuentra positiva y significativamente relacionado con la satisfacción con la vida de los adolescentes. En cuanto a las variables que constituyen la inteligencia emocional, la evaluación de las emocio- nes propias y la evaluación de las emociones de otros aparecen negativamente relacionadas con la resiliencia, el uso de las emociones presenta una relación directa con la influencia de la familia para evitar el consumo de drogas de los adolescentes, y el control emocional se relaciona directamente y de forma positiva con la satisfacción con la vida. Finalmente, todas las relaciones estadísticamente significativas entre la resiliencia, la satisfacción con la vida y las actitudes hacia el consumo de drogas de los adolescentes son negativas.

Así pues, tal como se expone en la Figura 2, la autoesti$\mathrm{ma}$, el apoyo social y la inteligencia emocional, muestran una capacidad predictiva del $4.4 \%$ de la resiliencia de los adolescentes, siendo la variable de mayor predicción el apoyo de la escuela $(\beta=-.136)$, aunque en sentido negativo. Con respecto a la satisfacción con la vida de los adolescentes, predicha en un $20.8 \%$ en este modelo, las variables con mayor capacidad predictiva han sido: la autoestima $(\beta=.190)$, el apoyo social de la familia $(\beta=.246)$, el control emocional $(\beta=$ .168), y la resiliencia $(\beta=-.095)$, ésta última en negativo. En cuanto a las actitudes de los adolescentes hacia el consumo de drogas, la resiliencia predice "el consumo de drogas no perjudica la salud" $(\beta=-.169)$, la "presión social para el consumo de drogas" ( $\beta=-.145)$ y los "efectos placenteros del consumo de drogas" $(\beta=-.134)$. Finalmente, el uso de la emoción muestra capacidad predictiva sobre la "influencia de la familia para evitar el consumo de drogas" $(\beta=.201)$. 


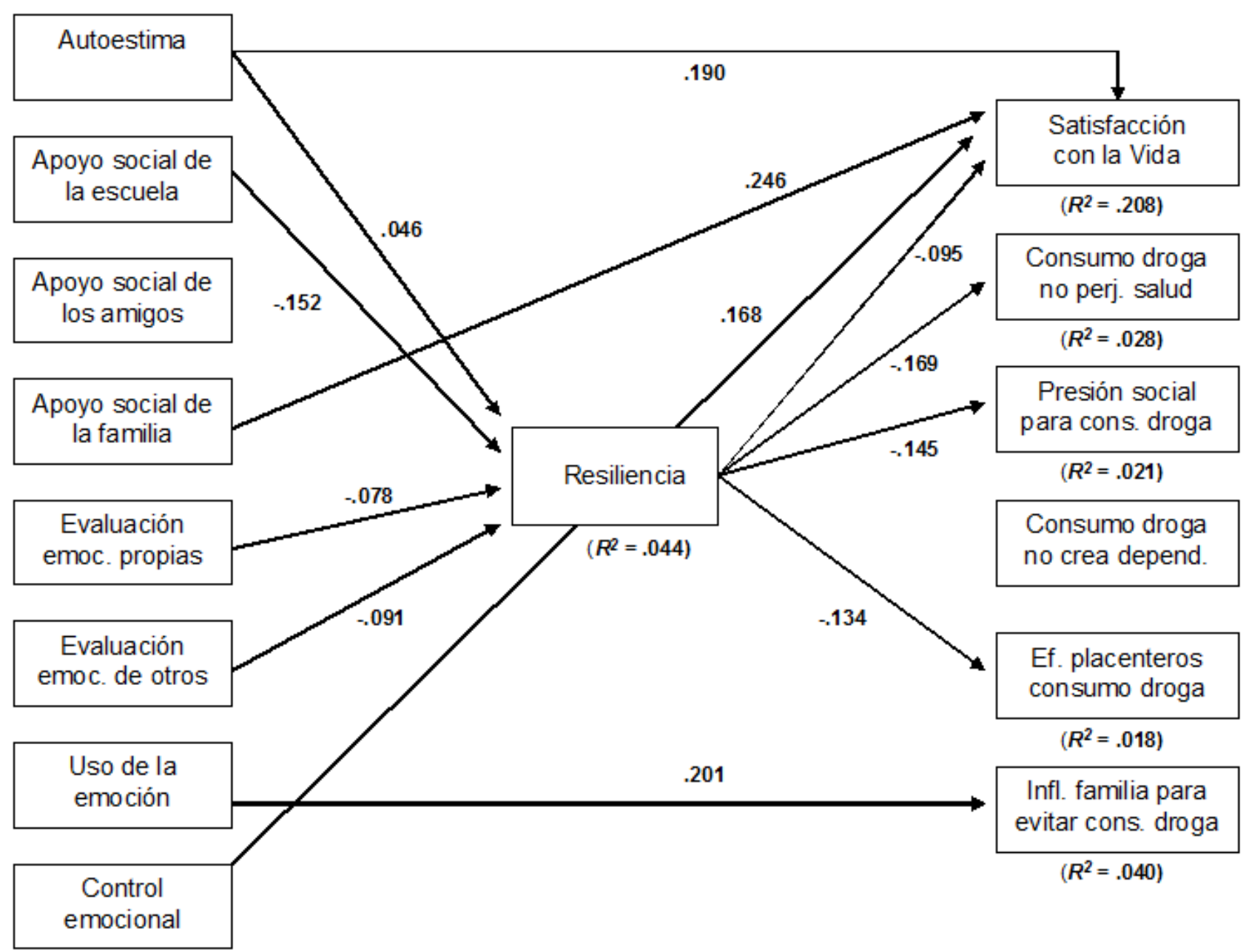

Nota: Todas las relaciones estructurales representadas son estadísticamente significativas $(p<.05)$. Por razones de claridad expositiva, no se muestran las correlaciones entre las variables exógenas ni los errores.

Figura 2. Solución estandarizada para el modelo propuesto de las relaciones entre autoestima, apoyo social, inteligencia emocional, resiliencia, satisfacción con la vida y actitudes de los adolescentes hacia el consumo de drogas.

\section{Discusión}

El objetivo principal de este trabajo ha consistido en la aplicación de ecuaciones estructurales para predecir la satisfacción con la vida de los adolescentes y sus actitudes hacia el consumo de drogas, a partir de la autoestima, el apoyo social, la inteligencia emocional y la resiliencia, haciendo esta última de variable mediadora. Hipotetizamos que la autoestima, el apoyo social y la inteligencia emocional estarían relacionadas con la resiliencia de los adolescentes; que la resiliencia se relacionaría con la satisfacción con la vida y con las actitudes hacia el consumo de drogas; y que las actitudes hacia las drogas y la satisfacción con la vida correlacionarían en sentido negativo.

A la vista de los resultados obtenidos cabe destacar, en primer lugar, que las características psicométricas de las escalas utilizadas han mostrando una apropiada adecuación a la muestra estudiada. Además, en cuanto a la fiabilidad de los instrumentos empleados, los coeficientes de consistencia interna han resultado satisfactorios para todos los factores, mostrando valores de alfa entre .705 y .809 . Ésta puede considerarse una primera aportación, dada la escasa existencia de instrumentos de evaluación validados actualmente en Angola.

Con respecto al cumplimiento de las hipótesis planteadas, cabe señalar que no se ha cumplido la tercera hipótesis en el sentido esperado, puesto que no ha resultado significativa ninguna de las relaciones encontradas entre satisfacción con la vida de los adolescentes y sus actitudes hacia el consumo de drogas. Sobre este aspecto, Zullig et al. (2001) encontraron relaciones entre la insatisfacción con la vida y el uso de sustancias adictivas, aunque no pudieron explicar si los adolescentes participaban en conductas de abuso de sustancias por su insatisfacción con la vida, o si estaban insatisfechos 
con la vida debido al abuso de sustancias, dejando esta cuestión como propuesta para futuras investigaciones. La diferencia de resultados entre el trabajo de Zullig y el nuestro puede deberse tanto al empleo de diferentes escalas de satisfacción con la vida, como a la forma de recoger los datos relacionados con el abuso de sustancias adictivas; en aquél mediante manifestación de consumo real y en éste a partir de actitudes hacia el consumo.

En cuanto a la primera hipótesis, que suponía el efecto directo de la autoestima, la inteligencia emocional y el apoyo social sobre la resiliencia de los adolescentes, se ha visto cumplida en parte, ya que de los ocho factores que cabía esperar que se relacionasen con la resiliencia, únicamente la autoestima presenta un efecto directo y positivo, mientras que los efectos del apoyo social de la escuela, la evaluación de las emociones propias y la evaluación de las emociones de otros sobre la resiliencia son negativos. A pesar de que Cunningham y Swanson (2010) refirieron que un elevado nivel de autoestima de los adolescentes, el apoyo social de los padres y el apoyo o asesoramiento de algún profesor de la institución educativa eran los mejores recursos para la construcción de la resiliencia ante los eventos estresantes y problemas de conducta, los resultados con nuestra muestra de estudio no se manifiestan en este sentido. Es más, los datos indican que cuanto mayor es el apoyo de la escuela, menor es el nivel de resiliencia de los adolescentes, no influyendo en ello ni el apoyo de los amigos ni el de la familia. Por otro lado, también cabe destacar el efecto directo de la autoestima, el apoyo social de la familia y el control emocional sobre la satisfacción con la vida, y el efecto también directo del uso de la emoción sobre la influencia de la familia para evitar el consumo de drogas, resultados en línea con los aportados por la literatura tal como más adelante especificaremos.

En relación con la segunda hipótesis, según la cual se esperaba que la resiliencia se relacionara de forma positiva con la satisfacción con la vida, los resultados han mostrado lo contrario, puesto que la relación entre ambas variables ha sido negativa. Según esto, cabría pensar que los adolescentes resilientes han conseguido construir tal recurso personal, pero eso no les lleva necesariamente a estar satisfechos con su vida. Sí se ha cumplido, en mayor medida, la previsión de la relación negativa entre la resiliencia y las actitudes hacia el consumo de drogas de los adolescentes, tal como ya habían anunciado Becoña et al. (2006), Boyce et al. (2002), o Sapienza y Pedromônico (2005).

Llegados a este punto cabe hacer algunas consideraciones. Por un lado, las bajas puntuaciones en resiliencia manifestadas por la muestra de adolescentes; las escasas relaciones entre autoestima, apoyo social e inteligencia emocional con la resiliencia; y el hecho de aparecer efectos directos de algunas de estas variables sobre la satisfacción con la vida y las actitudes hacia el consumo drogas de los adolescentes, induce a pensar que la resiliencia no cumple el papel de variable mediadora con el peso que se le había supuesto (Bonanno, 2012). Sin embargo, el hecho de que la resiliencia sí presente efectos sobre la satisfacción con la vida y las actitu- des hacia el consumo de drogas, parece indicar la importancia de su presencia en este modelo explicativo. Ahora bien, queda por resolver si resulta apropiado considerarla como variable mediadora o por el contrario incorporarla en el paquete de recursos personales de los adolescentes, situarla al mismo nivel que el resto de las variables, y a partir de ahí estudiar las relaciones existentes con la satisfacción con la vida y las actitudes hacia las drogas. Una cuestión más que queda por resolver es la dirección de las relaciones entre satisfacción con la vida y actitudes hacia el consumo de drogas, tal como planteaban Zullic et al. (2001), debido a la falta de relaciones encontradas en el presente trabajo.

No obstante, a pesar de no haberse cumplido completamente el modelo teórico que habíamos hipotetizado, no por ello dejan de tener importancia los resultados aquí obtenidos, pudiendo destacar diversas coincidencias con los encontrados en otros trabajos. Así por ejemplo, la relación positiva entre la autoestima y la resiliencia (Alfonso et al., 2001; Buckner et al., 2003; Gordon et al., 1998; Kumpfer, 1999), el efecto directo de la autoestima sobre la satisfacción con la vida (Byrne y Mazanov, 2001; Veselska et al., 2009), el efecto del apoyo de la familia sobre la satisfacción con la vida de los adolescentes (Ben-Ari y Gil, 2004; Bokhorst et al., 2010; Feitosa et al., 2005; Overle et al., 2011; Sánchez y Barrón, 2003), o el efecto directo del control emocional sobre la satisfacción con la vida (Austin et al., 2005; Schutte et al., 2010). Por otro lado, también coinciden nuestros resultados, en los que se pone de manifiesto la relación negativa entre la resiliencia y las actitudes en favor del consumo de drogas de los adolescentes, con los obtenidos por Becoña et al. (2006).

Desde una perspectiva aplicada, y en el marco de la psicología positiva, estos resultados pueden ser valiosos y servir de base para desarrollar estrategias de intervención encaminadas a prevenir el consumo de sustancias adictivas por parte de los adolescentes y favorecer su bienestar y crecimiento positivo (Fergus y Zimmerman, 2005; Veselska et al., 2009), poniendo énfasis en las variables que mayor explicación han aportado y teniendo en cuenta la dirección en que cada una de ellas debe plantearse. De cara a la prevención del consumo de sustancias, la resiliencia puede ser una importante vía para mejorar el comportamiento y los procesos internos de los individuos para que éstos puedan afrontar mejor todo tipo de situaciones relacionadas con el consumo de drogas.

A pesar de que en este trabajo no se hayan encontrado relaciones significativas entre la satisfacción con la vida de los adolescentes y las actitudes hacia el consumo de drogas, la literatura existente invita a continuar investigando en este sentido, por lo valioso de los objetivos perseguidos en favor de una mayor calidad de vida de niños y jóvenes. Adicionalmente, este trabajo ha tratado de dar respuesta a las propuestas de Lyubomirsky et al. (2005), Gadermann et al. (2010) y Sun y Shek (2012), que demandaban una mayor atención al estudio de la adolescencia, teniendo en cuenta que las relaciones entre estas variables, tradicionalmente, habían sido estudiadas en población adulta. Otra aportación relevante de este trabajo ha sido la propuesta de un modelo multidimen- 
sional. Aunque ya se había observado la importancia de estas variables en relación con la satisfacción con la vida (Chu et al., 2010) y el consumo de tabaco y otras sustancias adictivas (Downey et al., 2010; García del Castillo et al., 2012; Kokkevi et al., 2007), aquí hemos abordado su análisis de forma conjunta (path análisis).

En todo caso, los resultados de este trabajo deben ser considerados con precaución, dada la falta de estudios existentes en Angola sobre esta temática, lo cual imposibilita el contraste con los resultados obtenidos por otros investigadores en ese contexto cultural. No obstante, esto no invalida la importancia de este estudio como punto de partida para nuevas investigaciones. Actualmente, hay una escasez de información sobre las características del ambiente cultural de Angola y faltan estudios que contemplen las diferentes variables que podrían estar influyendo sobre los resultados, aparte de las contempladas en el modelo aquí sometido a verificación. Por ello, es deseable que nuevos trabajos vengan a ratificar o negar las conclusiones a las que ahora hemos llegado.

Como la mayoría de trabajos, éste también presenta sus limitaciones. En tal sentido, aunque el tamaño de la muestra es amplio, los datos fueron obtenidos a través de colabora-

\section{Referencias}

Alfonso, J. P., Huedo, T. B. y Espada, J. P. (2009). Factores de riesgo predictores del patrón de consumo de drogas durante la adolescencia. Anales de Psicología, 25(2), 330-338.

Alvarado, M. y Ricard, R. J. (2013). Developmental assets and ethnic identity as predictors of thriving in Hispanic adolescents. Hispanic Journal of Behavioral Sciences, 35(4), 510-523.

Austin, E. J., Saklofske, D. H. y Egan, V. (2005). Personality, well-being and health correlates of trait emotional intelligence. Personality and Individual Differences, 38, 547-558.

Avanci, J. Q., Assis, S .G., Santos, N. C. y Oliveira, R. V. (2007). Adaptação transcultural da Escala de Auto-Estima para adolescentes. Psicologia: Reflexâo e Crítica, 20(3), 397-405.

Becoña, E., Mínguez, M. C., López, A., Vázquez, M. J. y Lorenzo, M. C. (2006). Resiliencia y consumo de alcohol en jóvenes. Saludy drogas, 6(1), 89-111.

Ben-Ari, A. y Gil, S. (2004). Well-being among minority students: The role of perceived social support. Journal of Social work, 4(2), 215-225.

Bentler, P. M. (1990). Comparative fit indices in structural models. Psychological Bulletin, 107, 238-246.

Bentler, P. M. y Wu, E. J. C. (1995). EQS for Macintosh user's guide. Encino, CA: Multivariate Software.

Bokhorst, C. L., Sumter, S. R. y Westenberg, P. M. (2010). Social support from parents, friends, classmates, and teachers in children and adolescents aged 9 to 18 years: Who is perceived as most supportive? Social development, 19(2), 270-284.

Bollen, K. A. (1989). Structural equation modelling with latent variables. New York: Wiley.

Bonanno, G. A. (2012). Uses and abuses of the resilience construct: Loss, trauma, and health-related adversities. Social Sciences and Medicine, 74, 753-756.

Boyce Rodgers, K. y Rose, H. A. (2002). Risk and resiliency factors among adolescents who experience marital transitions. Journal of Marriage and Family, 64, 1024-1037.

Branden, N. (2000). Auto-estima: Como aprender a gostar de si mesmo. São Paulo, SP: Saraiva.

Braverman, M. T. (1999). Research on resilience and its implications for tobacco prevention. Nicotine \& Tobacco Research, 1(supl. 1), 67-72. dores (muestra por conveniencia), y recogidos mediante autoinformes de los adolescentes, lo cual puede incorporar un cierto grado de deseabilidad social. Además, los datos utilizados en este estudio son transversales, por lo que no se pueden establecer conclusiones fundamentadas en relaciones causa-efecto. Un diseño longitudinal podría reforzar el estudio y proporcionar mayor fiabilidad a los resultados y conclusiones. Es importante también señalar que el modelo aquí sometido a comprobación mediante ecuaciones estructurales es sólo uno de los posibles, existiendo otros modelos que podrían contribuir con nuevas explicaciones a la interpretación de las relaciones entre las variables estudiadas. Por otro lado, a la vista de los resultados obtenidos en este trabajo, se sugiere incluir en la muestra de estudio grupos de adolescentes susceptibles de haber padecido eventos estresantes, de tal forma que puedan encontrarse más próximos a una consideración real de la resiliencia. Finalmente, se sugiere continuar sometiendo a nuevas validaciones el Cuestionario de actitudes de los adolescentes hacia el consumo de drogas, e introducir modificaciones en el instrumento procurando la redacción de items en positivo, debido al efecto de método que pueden estar generando los items invertidos.
Brody, G. H. y Ge, X. (2001). Linking parenting processes and selfregulation to psychological functioning and alcohol use during early adolescence. Journal of Family Psychology, 15(1), 82-94.

Buckner, J. C., Mezzacappa, E. y Beardslee, W. R. (2003). Characteristics of resilient youths living in poverty: The role of self-regulatory processes. Development and Psychopathology, 15, 139-162.

Buelga, S., Cava, M. J. y Musitu, G. (2012). Reputación social, ajuste psicosocial y victimización entre adolescentes en el contexto escolar. Anales de Psicología, 28(1), 180-187.

Byrne D. G. y Mazanov, J. (2001). Self-esteem, stress and cigarette smoking in adolescents. Stress Health, 17, 105-110.

Chu, P. S., Saucier, D. A. y Hafner, E. (2010). Meta-analysis of the relationships between social support and well-being in children and adolescents. Journal of Social and Clinical Psychology, 29(6), 624-645.

Ciarrochi, J., Scott, G., Deane, F. P. y Heaven, P. C. (2003). Relations between social and emotional competence and mental health: A construct validation study. Personality and Individual Differences, 35, 1947-1963.

Cohen, S. (2004). Social relationships and health. American Psychologist, 59, 676-684.

Cohen, S. y Wills, T. A. (1985). Stress, social support, and the buffering hypothesis. Psychological Bulletin, 98, 310-357.

Clara, I.P., Cox, B.J., Enns, M.W., Murray, L.T. y Torgrudc, L.J. (2003). Confirmatory Factor Analysis of The Multidimensional Scale of Perceived Social Support in clinically distressed and student samples. Journal of Personality Assessment, 81(3), 265-270.

Cook, T. D., Herman, M. R., Phillips, M. y Settersten, R. A. (2002). Some ways in which neighborhoods, nuclear families, friendship groups, and schools jointly affect changes in early adolescent development. Child Development, 73, 1283-1309.

Cunningham, M. y Swanson, D.P. (2010). Educational resilience in African American adolescents. The Journal of Negro Education, 79(4), 473-487.

Demaray, M. K. y Malecki, C. K. (2002). The relationship between perceived social support and maladjustment for students at risk. Psychology in the Schools, 39, 305-316.

Diener, E. (2000). Subjective well-being: The science of happiness and a proposal for a national index. American Psychologist, 55, 34-43.

Downey, L.A., Johnston, P.J., Hansen, K., Birney, J. y Stough, C. (2010). Investigating the mediating effects of emotional intelligence and coping 
on problem behaviours in adolescents. Australian Journal of Psychology, 62, 20-29.

Elmore, G.M. y Huebner, E.S. (2010). Adolescents' satisfaction with school experiences: Relationships with demographics, attachment relationships, and school engagement behavior. Psychology in the Schools, 47(6), 525-537.

Escámez, J. y García, R. (1990). Actitudes de los alumnos de E.G.B. ante las drogas. En J. Escámez et al. (Eds.), Drogas y escuela. Una propuesta de prevención (pp. 105-119). Madrid: Dykinson

Feitosa, F., de Matos, M., del Prette, Z. y del Prette, A. (2005). Suporte social, nível socio-economico e o ajustamento social e escolar de adolescentes portugueses. Temas em Psicología, 13, 129-138.

Fergus, S. y Zimmerman, M.A. (2005). Adolescent resilience: A framework for understanding healthy development in the face of risk. Annual Review of Public Health, 26, 399-419.

Finney, S. J. y DiStefano, C. (2006). Non-normal and categorical data in SEM. En G. R. Hancock y R. O. Mueller (Eds.), Structural Equation Modeling: A second course (pp. 269-314). Greenwich, CO: Information Age Publishing.

Gadermann, A. M., Schonert-Reichl, K. A. y Zumbo, B. D. (2010). Investigating validity evidence of the Satisfaction with Life Scale adapted for children. Social Indicators Research, 96, 229-247.

García del Castillo, J. A., Dias, P., Díaz-Pérez, J. y García del CastilloLópez, A. (2012). The mediating role of self-regulation in cigarette smoking and alcohol use among young people. Anales de Psicología, 28(1), 1-10.

García del Castillo, J. A. y Dias, P. (2007). Análisis relacional entre los factores de protección, resiliencia, autorregulación y consumo de drogas. Saludy Drogas, 7(2), 309-332.

Garnefski, N. y Diekstra, R. F. W. (1996). Perceived social support from family, school and peers: Relationship with emotional and behavioral problems among adolescents. Journal of the American Academy of Child and Adolescent Psychiatry, 35, 1657-1664.

Gordon Rouse, K. A., Ingersoll, G. M. y Orr, D. P. (1998). Longitudinal health endangering behavior risk among resilient and nonresilient early adolescents. Journal of Adolescent Health, 23, 297-302.

Gutiérrez, M. y Gonçalves, T. O. (2013). Activos para el desarrollo, ajuste escolar y bienestar subjetivo de los adolescentes. International Journal of Psychology and Psychological Therapy, 13, 339-355.

Haggerty, R. J., Sherrod, L. R., Gamezy, N. y Rutter, M. (2000). Stress, risk and resilience in children and adolescents: process, mechanisms and interventions. New York: Cambridge University Press.

Henrich, G. y Herschbach, P. (2000).Questions on life satisfaction (FLZ $\left.{ }^{M}\right)$. A short questionnaire for assessing subjective quality of life. European Journal of Social Psychology, 16, 150-159.

Ho, M. Y, Cheung, F. M. y Cheung, S. F. (2010). The role of meaning in life and optimism in promoting well-being. Personality and Individual Differences, $48,658-663$.

Hu, L. y Bentler, P. M. (1999). Cut-off criteria for fit indexes in covariance structure analysis: conventional criteria versus new alternatives. Structural Equation Modeling, 6, 1-55.

Kaplan, D. (2000). Structural equation modeling: Foundations and extensions. Thousand Oaks, CA: Sage.

Kokkevi, A., Richardson, C., Florescu, S., Kuzman, M. y Stergar, E. (2007). Psychosocial correlates of substance use in adolescence: A crossnational study in six European countries. Drug and Alcohol Dependence, $86,67-74$.

Kumpfer, K. L. (1999). Factors and processes contributing to resilience: The resilience framework. En M. D. Glantz y J. L. Johnson (Eds.), Resiliency and development: Positive life adaptations (pp. 179-224). New York: Kluwer Academic.

Lent, R. W., Singley, D., Sheu, H. B., Gainor, K. A., Brenner, B. R., Treistman, D., et al. (2005). Social cognitive predictors of domain and life satisfaction: Exploring the theoretical precursors of subjective wellbeing. Journal of Consulting Psychology, 52(3), 429-442.

Liu, Y., Wang, Z. y Lü, W. (2013). Resilience and affect balance as mediators between trait emotional intelligence and life satisfaction. Personality and Individual Differences, 54, 850-855.
López, S. y Rodríguez-Arias, J. L. (2010). Factores de riesgo y de protección en el consumo de drogas en adolescentes y diferencias según edad y sexo. Psicothema, 22, 4, 568-573.

Lyubomirsky, S., King, L. y Diener, E. (2005). The benefits of frequent positive affect: Does happiness lead to success? Psychological Bulletin, 131, 803-855

Mann, M., Hosman, C. M., Schaalma, H. P. y de Vries, N. K. (2004). Selfesteem in a broad-spectrum approach for mental health promotion. Health Education Research, 19, 357-372.

Marques, S., Pais-Ribeiro J. y Lopez, S. (2007). Validation of a Portuguese version of the Students'Life Satisfaction Scale. Applied Research in Quality of Life, 2, 83-94.

Mayer, J.D., Salovey, P. y Caruso, D.R. (2008). Emotional intelligence. New ability or eclectic traits? American Psychologist, 63, 503-517.

Oliva, A., Antolín, L. y López, A. M. (2012). Development and validation of a scale for the measurement of adolescents' developmental assets in the neighborhood. Social Indicators Research, 106, 563-576.

Oliva, A., Ríos, M., Antolín, L., Parra. A., Hernando, A. y Pertegal, M. A. (2010). Más allá del déficit: Construyendo un modelo de desarrollo positivo adolescente. Infancia y Aprendizaje, 33, 223-234.

Oliver, A., Sancho, P., Galiana, L., Tomás, J. M. y Gutiérrez, M. (2012). Efecto de método asociado a los ítems en negativo en la versión en portugués de la escala de autoestima de Rosenberg. Revista Iberoamericana de Diagnóstico y Evaluación Psicológica, 33, 75-91.

Ortega, P., Mínguez, R. y Pagán, F.A. (1993). Actitudes hacia la droga en los alumnos de E.G.B. de la Región Murciana. Anales de Psicología, 9(1), 7-18

Overle, E., Schonert-Reichl, K. A. y Zumbo, B. D. (2011). Life satisfaction in early adolescence: Personal, neighborhood, school, family, and peer influences. Journal of Youth Adolescence, 40, 889-901.

Panadero, S. y Muñoz, M. (2014). Salud, calidad de vida y consumo de sustancias en función del tiempo en situación sin hogar. Anales de Psicologia, 3(1) 70-77.

Pavot, W. y Diener, E. (1993). Review of the Satisfaction with Life Scale. Psychological Assessment, 5(2), 164-172.

Pavot, W. y Diener, E. (2008). The Satisfaction with Life Scale and the emerging construct of life satisfaction. The Journal of Positive Psychology, 3(2), 137-152.

Pérez, M. y Lucio-Gómez, E. (2010). Construcción de un modelo de riesgo en el consumo de alcohol y otras sustancias ilícitas en adolescentes estudiantes de bachillerato. Health and Addictions/Salud y Drogas, 10 (1) 79-96.

Petrides, K. V., Frederickson, N. y Furnham R. (2004). The role of trait emotional intelligence in academic performance and deviant behaviour at school. Personality and Individual Differences, 36, 277-293.

Ramaswamy, V., Aroian, K.J. y Templin, T. (2009). Adaptation and psychometric evaluation of the Multidimensional Scale of Perceived Social Support for Arab American adolescents. American Journal of Community Psychology, 43, 49-56.

Richardson, C. G., Kwon, J. Y. y Ratner, P. A. (2013). Self-esteem and the initiation of substance use among adolescents. Canadian Journal of Public Health, 104(1), e60-e63.

Rodríguez, A. y Goñi, A. (2011). La estructura tridimensional del bienestar subjetivo. Anales de Psicología, 27(2), 327-332.

Rosenberg, M. (1965). Society and the adolescent self-image. New Jersey, USA: Princeton University Press.

Ruiz, F. y Ruiz-Risueño, J. (2011). Variables predictoras de consumo de alcohol entre adolescentes españoles Anales de Psicología, 2(2), 350-359.

Salamó, A, Gras, M. E. y Font-Mayolas, S. (2010). Patrones de consumo de alcohol en la adolescencia. Psicothema, 22, 189-195.

Sánchez, E. y Barrón, A. (2003). Social psychology of mental health: The social structure and personality perspective. The Spanish Journal of Psychology, 6, 3-11

Santos, P. J. y Maia, J. (2003). Análise factorial confirmatória e validaçao preiminar de uma versao portuguesa da Escala de Auto-estima de Rosenberg. Psicología: Teoria, Investigaçao e Prática, 2, 253-268.

Sapienza, G. y Pedromônico, M.R.M. (2005). Risco, proteção e resiliência no desenvolvimento da criança e do adolescente. Psicologia em Estudo, 10(2), 209-216. 
Schmitt, D. P. y Allik, J. (2005) Simultaneous administration of the Rosenberg Self-Esteem Scale in 53 nations: Exploring the universal and culture-specific features of global self-esteem. Journal of Personality and Social Psychology, 89, 632-642.

Schutte, N. S., Thorsteinsson, E. B., Hine, D. W., Foster, R., Cauchi, A. y Binns, C. (2010). Experiential and rational processing styles, emotional intelligence and wellbeing. Australian Journal of Psychology, 62, 14-19.

Seligman, M .E .P. y Csikszentmihalyi, N. (2000). Positive psychology: An introduction. American Psychologist, 55, 5-14.

Sheldon, K. M. y King, L. (2001). Why positive psychology is necessary? American Psychologist, 56, 216-217.

Simons, J. S. y Carey, K. B. (2002). Risk and vulnerability for marijuana use problems: The role of affect dysregulation. Psychology of Addictive Behaviors, 16, 72-75.

Skaar, N. R. y Williams, J. E. (2012). Emotional intelligence as a predictor of adolescent risk behaviour participation and perception. I-manager's Journal on Educational Psychology, 5(4), 32-47.

Smith, B.W., Dalen, J., Wiggins, K., Tooley, E., Christopher, P. y Bernard, J. (2008). The Brief Resilience Scale: Assessing the ability to bounce back. International Journal of Behavioral Medicine, 15, 194-200.

Sun, R. C. y Shek, D. T. (2010). Life satisfaction, positive youth development, and problem behaviour among Chinese adolescents in Hong Kong. Social Indicators Research, 95, 455-474.

Sun, R. C. y Shek, D. T. (2012). Positive youth development, life satisfaction and problem behaviour among Chinese adolescents in Hong Kong: A replication. Social Indicators Research, 105(3), 541-559.

Terry, T. y Huebner, S. (1995). The relationship between self-concept and life satisfaction in children. Social Indicators Research, 35, 39-52.

Tomás, J. M. y Oliver, A. (1999). Rosenberg's self-esteem scale: Two factors or method effects? Structural Equation Modeling, 6(1), 84-98.

Valois, R. E., Zullig, K. J., Huebner, E. S. y Drane, J. W. (2001). Relationship between life satisfaction and violent behaviours among adolescents. American Journal of Health Behavior, 25, 353-366.

Veselska, Z., Geckova, A. M., Orosova, O., Gajdosova, B., van Dijk, J. P. y Reijneveld, S. A. (2009). Self-esteem and resilience: The connection with risky behavior among adolescents. Addictive Behaviors, 34, 287-291.
Videra, A. y Reigal, R. (2013). Autoconcepto físico, percepción de salud y satisfacción vital en una muestra de adolescentes. Anales de Psicología, 29(1), 141-147.

WHOQOL Group (1995). The World Health Organization Quality of Life Assessment (WHOQOL): Position paper from the World Health Organization. Social Science \& Medicine, 41, 1403-1409.

Wild, L. G., Flisher, A. J., Bhana, A. y Lombard, C. (2004). Associations among adolescent risk behaviours and self-esteem in six domains. Journal of Child Psychology and Psychiatry, 45, 1454-1467.

Wills, T. A., Walker, C., Mendoza, D. y Ainette, M. G. (2006). Behavioral and emotional self-control: Relations to substance use in samples of middle and high school students. Psychology of Addictive Behaviors, 20(3), 265-278.

Wills, T. A. y Dishion, T. J. (2004). Temperament and adolescent substance use: A transactional analysis of emerging self-control. Journal of Clinical Child and Adolescent Psychology, 33, 69-81.

Wills, T. A. y Stoolmiller, M. (2002). The role of self-control in early escalation of substance use: A time-varying analysis. Journal of Consulting and Clinical Psychology, 70, 986-997.

Wong, C. S. y Law, K. S. (2002). The effects of leader and follower emotional intelligence on performance and attitude: An exploratory study. The Leadership Quarterly, 13, 243-274.

Wood, A. M., Froh, J. J. y Geraghty, A.W. (2010). Gratitude and well-being: A review and theoretical integration. Clinical Psychology Review, 30(7), 890-905.

Zimet, G. D., Dahlem, N. W., Zimet, S. G. y Farley, G. K. (1988). The multidimensional scale of perceived social support. Journal of Personality Assessment, 52, 30-41.

Zullig, K. J., Valois, R. F., Huebner, E. S., Oeltmann, J. E. y Drane, W. J. (2001). Relationship between perceived life satisfaction and adolescent substance abuse. Journal of Adolescent Health, 29, 279-288.

(Artículo recibido: 17-4-2012; revisado: 6-10-2013; aceptado: 19-2-2014) 